4. C.Domb and M. F.Sykes, Proc. Phys. Soc., (London) B69 (1956) 486.

5. G.S. Rushbrooke and P. J. Wood, Mol. Phys. 1 (1958) 257.

6. C. Domb and D.W.Wood, Proc. Phys. Soc. 86 (1965)

1.
7. C. Domb, Phil. Mag. Suppl. 9 (1960) nos. 34, 35.

8. G. A. Baker Jr., H. E. Gilbert, J. Eve and G.S. Rushbrooke, Phys. Letters 22 (1966) 269.

\title{
THE $\lambda$-POINT, ENTROPY AND DENSITY OF ROTATING HeII
}

\author{
F. POBELL, W. SCHOEPE and W. VEITH \\ Physik Department der Technischen Hochschule München, Germany
}

Received 17 July 1967 The $\lambda$-point, entropy and density of $\mathrm{He}^{\mathrm{II}}$ have been found to be independent of rotation up to $\omega=110 \mathrm{sec}^{-1}$
to within $\Delta T_{\lambda}=10^{-5} \mathrm{o}_{\mathrm{K}}, \Delta S=7 \times 10^{-4}$ joule/mole ${ }^{\circ} \mathrm{K}$, and $\Delta \rho / \rho=2 \times 10^{-6}$ respectively.

Recently Andronikashvili and Tsakadze [1] reported that the density of liquid helium increased in an anomalous and temperature dependent way when the liquid was set into rotation. The relative increase $(\rho(\omega)-\rho(0)) / \rho(0)$ for $\omega=$ $=30 \mathrm{sec}^{-1}$ amounted to $2.1 \times 10^{-4}$ near below the $\lambda$-point and dropped to zero within $5 \mathrm{mdeg}$ in the immediate vicinity of the $\lambda$-point, remaining zero for $T>T_{\lambda}$.

If one assumes the $\lambda$-transition of rotating helium to be of the second order the finite density change described above may lead to a finite shift of the $\lambda$-temperature. We have therefore measured the temperature of the $\lambda$-point for nonrotating and rotating helium up to angular velocities of $\omega=110 \mathrm{sec}^{-1}$.

The liquid was rotated in a thermally insulated cylindrical copper can $(3.6 \mathrm{~cm}$ diam. and $6.2 \mathrm{~cm}$ long) with a carbon resistor as a thermometer immersed in the rotating liquid. The passing of the $\lambda$-point was recognized by an abrupt change of the heating rate and, independently, by a sudden overheating of the carbon resistor when its heat input exceeded $10^{-7}$ watt.

Our experiments which were performed at higher rotational speeds and with a higher precision than previous observations [2], show to an accuracy of $10^{-5} \mathrm{~K}$ that the temperature of the $\lambda$-point is independent of rotation up to an angular velocity of $\omega=110 \mathrm{sec}^{-1}$.

The change of the density at $\omega=30 \mathrm{sec}^{-1}$ and $T=1.74^{\circ} \mathrm{K}$ reported in [1] should lead to a change of entropy $\Delta S=S(\omega)-S(0)=0.1$

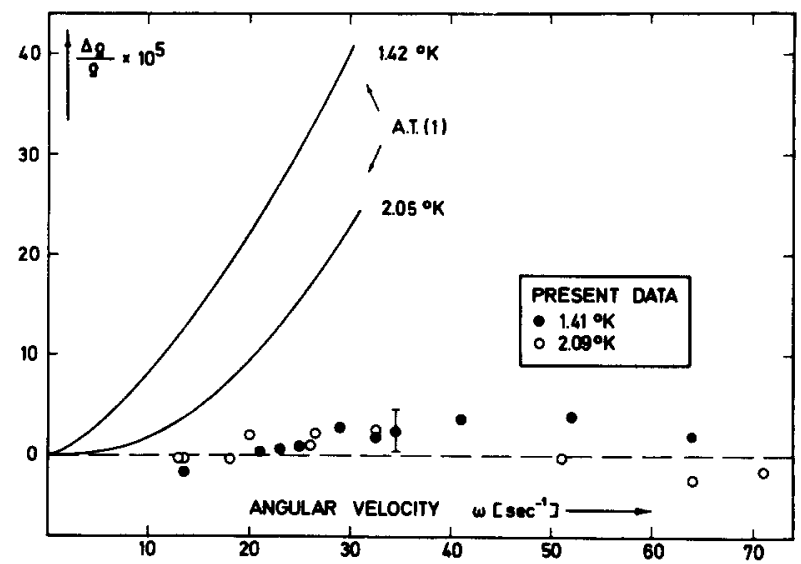

Fig. 1. Relative variation of the density of liquid helium with rotation. The solid curves represent the pycnometrie data of ref. 1. The open and solid points are our dielectric measurements at two similar temperatures. The temperature was controlled to better than $10^{-4} \mathrm{o}_{\mathrm{K}}$.

Joule/mole ${ }^{\circ} \mathrm{K}$ [2]. By setting $5 \mathrm{~g}$ of $\mathrm{He}^{\mathrm{II}}$ in rotation adiabatically we looked for a temperature change. Within $\Delta T=10^{-4} \mathrm{O} \mathrm{K}$ at $T=1.80^{\circ} \mathrm{K}$ we did not find any temperature changes caused by rotation up to $\omega=110 \mathrm{sec}^{-1}$. This means that the entropy remains constant within $\Delta S=7 \times 10^{-4}$ joule/mole ${ }^{0} \mathrm{~K}$ and that the density is not dependent on $\omega$ within $\Delta \rho / \rho=2 \times 10^{-6}$ at our angular velocities and at $T=1.80^{\circ} \mathrm{K}$.

These results can only be understood if the density of helium does not depend on rotation in 


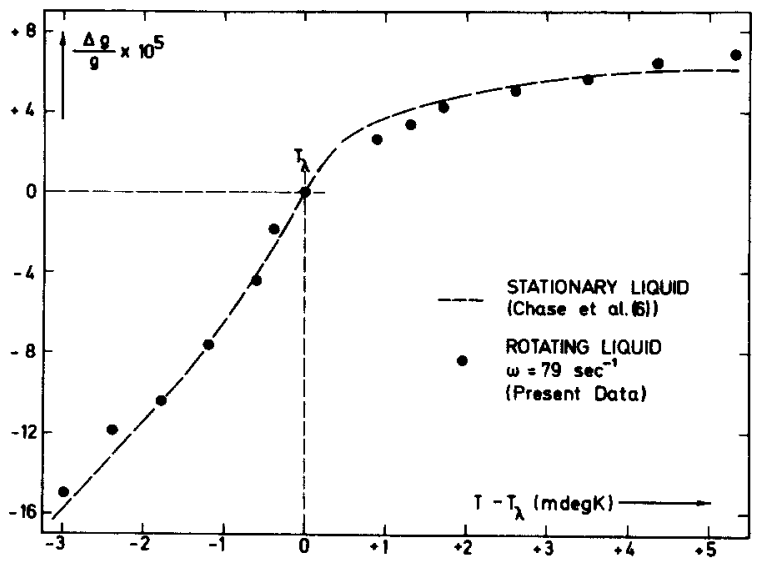

Fig. 2. Relative variation of the density with temperature of stationary helium (according to ref.6) and of rotating helium $\left(\omega=79 \mathbf{s e c}^{-1}\right)$. The points were taken over a time of 54 minutes between $2.10^{\circ} \mathrm{K}$ and $2.20^{\circ} \mathrm{K}$ with the helium warming up; they were fitted to the data of ref. 6 at $T_{\lambda}$.

the way reported in [1]. Recent experiments by Ahlers [2], Andelin [3] and by Smith et al. [4] did, in fact, show no indication of a density variation with rotation.

This conclusion is strongly supported by our own measurements of the liquid density shown in fig. 1. Evidently the density remains constant independent of $\omega$ in contrast to the results by Andronikashvili and Tsakadze [1]. For these experiments we used a dielectric method [5]: A cylinder capacitor $(2 \mathrm{~cm}$ long, $0.9 \mathrm{~cm}$ diam., $10^{-2} \mathrm{~cm}$ spacing) immersed vertically in the rotating liquid controlled the frequency of a $16 \mathrm{MHz}$ tunnel diode oscillator. By observing the fre- quency of the oscillator it was possible to detect small density changes of the liquid inside the capacitor. Fig. 2 shows, in addition, that within $\Delta \rho / \rho=5 \times 10^{-6}$ no anomalous drop of the density occurs at the $\lambda$-point when rotating superfluid helium is heated through the $\lambda$-temperature (while in ref. 1 a drop of $\Delta \rho / \rho=2.1 \times 10^{-4}$ was reported already for $\omega$ as small as $30 \mathrm{sec}^{-1}$ ). The same results were obtained with another cylinder capacitor having a spacing of $0.5 \mathrm{~mm}$.

Our results are in agreement with early experiments by Chase [7], who did not find any influence of rotation on the velocity of sound: The velocity of sound remained constant within $5 \times$ $\times 10^{-6}$ for angular velocities up to $\omega=20 \mathrm{sec}^{-1}$.

We wish to express our gratitude to Professor $\mathrm{K}$. Dransfeld for stimulating interest and support of the research. Thanks are due to Professor $\mathrm{H}$. Meyer for helpful advice concerning the tunnel diode oscillator and to Professor W. Brenig and Dr. Wagner for several useful discussions.

\section{References}

1. E.L.Andronikashvili and J.S.Tsakadze, Phys, Letters 18 (1965) 26; 20 (1966) 446; JETP Letters 2 (1965) 177.

2. G.Ahlers, Bull. Am. Phys, Soc, 12 (1967) 551.

3. J.Andelin, Phys. Rev. Letters 18 (1967) 483.

4. E.Smith, R. Walton, H.V. Bohm and J.D. Reppy, Phys. Rev. Letters 18 (1967) 637.

5. C. Boghosian, H. Meyer and J.E.Rives, Phys. Rev. 146 (1966) 110.

6. C.E.Chase, E. Maxwell, W. E. Millett, Physica 27 (1961) 1129.

7. C.E.Chase, J.Fineman and W.E. Millett, Physica 25 (1959) 631. 